\section{Creeping Bentgrass Seedling Tolerance to Herbicides and Paclobutrazol}

\author{
John E. Kaminski and Peter H. Dernoeden \\ Department of Natural Resource Sciences and Landscape Architecture, \\ University of Maryland, College Park, MD 20742
}

\author{
Cale A. Bigelow \\ Agronomy Department, Purdue University, West Lafayette, IN 47907
}

Additional index words. herbicide residues, phytotoxicity, renovation, bensulide, chlorsulfuron, ethofumesate, prodiamine, siduron

\begin{abstract}
The tolerance of creeping bentgrass (Agrostis stolonifera L.) seedlings to many herbicides has not been evaluated. Three field studies were conducted between fall and spring from 1998 to 2002 to assess creeping bentgrass seedling tolerance to five herbicides and paclobutrazol. The primary objectives of this investigation were to assess bentgrass tolerance to these chemicals when applied at various timings following seedling emergence, and establishment of new seedlings as influenced by potential soil residues in the spring following a fall application of the chemicals. Treatments were applied 2, 4, or 7 weeks after either 'Crenshaw' or ' $L-93$ ' creeping bentgrass seedlings had emerged. Siduron $\left(6.7\right.$ and $\left.9.0 \mathrm{~kg} \cdot \mathrm{ha}^{-1}\right)$ and bensulide $\left(8.4 \mathrm{~kg} \cdot \mathrm{ha}^{-1}\right)$ were noninjurious when applied two weeks after seedling emergence ( 2 WASE). Bensulide $\left(14 \mathrm{~kg} \cdot \mathrm{ha}^{-1}\right)$, ethofumesate $\left(0.84 \mathrm{~kg} \cdot \mathrm{ha}^{-1}\right)$, prodiamine $\left(0.36 \mathrm{~kg} \cdot \mathrm{ha}^{-1}\right)$ and paclobutrazol $\left(0.14 \mathrm{~kg} \cdot \mathrm{ha}^{-1}\right)$ were too injurious to apply 2 WASE, but they were generally safe to apply at 4 WASE. Chlorsulfuron $\left(0.14 \mathrm{~kg} \cdot \mathrm{ha}^{-1}\right)$ was extremely phytotoxic to seedlings when applied 2 WASE. Plots were treated with glyphosate and overseeded the following spring. The overwintering soil residuals of prodiamine and bensulide $\left(14.0 \mathrm{~kg} \cdot \mathrm{ha}^{-1}\right)$ unacceptably reduced spring establishment. All other herbicides and paclobutrazol had little or no adverse residual effects on spring establishment. Chemical names used: $N$-(phosphonomethyl)gycline (glyphosate); $( \pm)-\left(\mathbf{R}^{*}, \mathbf{R}^{*}\right)$-beta[(4-chlorophenyl)methyl]-alpha-(1,1-dimethylethyl)-1H-1,2,4-triazole-1-ethanol (paclobutrazol); 2-ethoxy-2,3-dihydro-3,3-dimethyl-5-benzofuranyl methanesulfonate (ethofumesate); S-(0,0-diisopropyl phosphorodithioate) ester of $\mathrm{N}$-(2-mercaptoethyl) benzenesulfonamide (bensulide); [1-(2-methylcyclohexyl)-3-phenylurea] (siduron); $\mathrm{N}^{3}, \mathrm{~N}^{3}$-di-n-propyl-2,4-dinitro-6-(trifluoromethyl)- $m$-phenylenediamine (prodiamine); 2chloro- $\mathrm{N}$-[(4-methoxy-6-methyl-1,3,5-triazin-2-yl)aminocarbonyl] benzenesulfonamide (chlorsulfuron).
\end{abstract}

The tolerance of creeping bentgrass seedlings to most available herbicides has not been evaluated in the transition zone climate of the mid-Atlantic region. This type of research is needed due to the increased use of creeping bentgrass as a fairway turf in the region. Late summer or early fall bentgrass establishment may be hampered by the presence of an existing large seed bank of annual bluegrass (Poa annua L. ssp. апnиa) and other weeds, which often out-compete creeping bentgrass seedlings during establishment. Ethofumesate can effectively control annual bluegrass postemergence (Branham, 1991; Dernoeden and Turner, 1988). Chlorsulfuron was shown to have postemergence activity on annual bluegrass without injuring mature bentgrass (Gaul and Christians, 1988; Maloy and Christians, 1986). Bensulide, prodiamine and other preemergence herbicides have been effective

Received for publication 7 Apr. 2003. Accepted for publication 6 Oct. 2003. Mention of trademark or proprietary product does not constitute a guarantee or warranty of the product by the University of Maryland or the authors and does not imply its approval to the exclusion of other products that also may be suitable. A contribution of the Maryland Agricultural Experiment Station.

${ }^{1}$ Corresponding author; e-mail pd9@umail.umd.edu.
Three studies were conducted between fall and spring from 1998 to 2002 to assess creeping bentgrass seedling tolerance to five herbicides and paclobutrazol and their overwintering soil residues. The primary objectives of this investigation were to assess the following: creeping bentgrass tolerance to five herbicides and paclobutrazol when applied at various timings following seedling emergence; bentgrass establishment rate in response to herbicide and paclobutrazol treatment; and establishment of new seedlings as influenced by potential overwintering chemical residues following re-seeding of fall-treated plots in the spring.

\section{Materials and Methods}

Field studies were initiated on mature stands of fairway height bentgrass turf at the University of Maryland Turfgrass Research Facilities in Silver Spring, Md. (1998), and College Park, Md. (2000 and 2001). Soil at the Silver Spring site was Sassafras sandy loam (fine-loamy, siliceous mesic Typic Hapludult) with a $\mathrm{pH}$ of 6.2 and $2.1 \%$ organic matter. In College Park, soil was a Keyport silt loam (fine, mixed, semiactive, mesic Aquic Hapludult) with a $\mathrm{pH}$ of 6.5 and $1.2 \%$ organic matter. Three separate bentgrass sites were treated with glyphosate $\left(1.1 \mathrm{~kg} \cdot \mathrm{ha}^{-1}\right)$ on 14 Sept. 1998, 11 Sept. 2000 and 20 Aug. 2001, respectively. The sites then were vertical cut (3.8 to $4.4 \mathrm{~cm}$ width by 1.3 to $2.5 \mathrm{~cm}$ depth) in three to five directions and seeded with a drop seeder at approximately $55 \mathrm{~kg} \cdot \mathrm{ha}^{-1}$ with 'Crenshaw' on 21 Sept. 1998 and 'L-93' on 18 Sept. 2000 and 7 Sept. 2001. All seeded sites were syringed frequently, and most seedlings emerged within six to eight days. Once seedlings emerged, the sites were irrigated as needed to avoid drought stress. At the time of seeding a starter $19 \mathrm{~N}-11 \mathrm{P}-4 \mathrm{~K}$ fertilizer (Contec, Scott's Co., Marysville, Ohio) was applied to supply $\mathrm{N}$ at $50 \mathrm{~kg} \cdot \mathrm{ha}^{-1}$ and an additional $50 \mathrm{~kg} \cdot \mathrm{ha}^{-1} \mathrm{~N}$ were applied $\approx 21 \mathrm{~d}$ after seedling emergence. The areas were mowed beginning two to three weeks after seedling emergence three times per week to a height of $\approx 1.8 \mathrm{~cm}$ using a walk-behind greens mower.

The chemicals evaluated and their formulations were as follows: bensulide $4 \mathrm{~L}$; chlorsulfuron 75DF; ethofumesate 1.5EC; siduron 3.1G (1998) and 50WP (2000 and 2001), prodiamine $65 \mathrm{DG}$; and paclobutrazol $2 \mathrm{SC}$. Rates and application dates of the chemicals evaluated are shown in the data tables. All rates chosen were based on label and local use recommendations. The prodiamine rate of 0.36 $\mathrm{kg} \cdot \mathrm{ha}^{-1}$ was chosen because previous studies showed that it was as effective as higher rates for controlling annual bluegrass in Maryland (Dernoeden, 1998). Treatments were applied two, four, or seven weeks after seedlings had emerged. Seedlings were in the three-leaf to two-tiller stage when each study was initiated. Granular products were applied with a shaker bottle and all other treatments were applied with a CO pressurized $(262 \mathrm{kPa})$ sprayer calibrated to deliver $467 \mathrm{~L}$ of water/ha. The test areas were irrigated with at least $2.0 \mathrm{~cm}$ of water within $24 \mathrm{~h}$ of each application. 
To evaluate the potential overwintering soil residual effects on spring seedling emergence from the fall applied herbicides and paclobutrazol, the test sites were treated with glyphosate $\left(1.1 \mathrm{~kg} \cdot \mathrm{ha}^{-1}\right)$ on $23 \mathrm{Apr}$. in 1999 and 2001 and on 2 May 2002. Each site was vertical cut and seeded as previously described with 'Crenshaw' creeping bentgrass on 6 May 1999 and 'L-93' creeping bentgrass on 1 May 2001 and 10 May 2002. The previously described fertilizer was applied at seeding to supply $\mathrm{N}$ at $50 \mathrm{~kg} \cdot \mathrm{ha}^{-1}$ and again with an additional 50 $\mathrm{kg} \cdot \mathrm{ha}^{-1} \mathrm{~N} \approx 21 \mathrm{~d}$ after emergence.

Plots measured $1.5 \times 1.5 \mathrm{~m}$ and were arranged in a randomized complete block design with four replications. Seedling injury was assessed using bentgrass cover and overall turfgrass quality ratings. Percent bentgrass cover was assessed visually using a $0 \%$ to $100 \%$ linear scale where $0=$ no bentgrass and 100 $=$ entire plot covered with bentgrass. Overall turfgrass quality was assessed visually on a 0 to 10 scale where $0=$ entire plot area brown or dead; 7.5 = minimum acceptable quality for a golf course fairway; and $10=$ optimum greenness and density. Only percent bentgrass cover ratings were used as a measure of potential adverse soil residues on establishment in the spring overseeding portion of the study. Safe treatments caused little or no reduction in bentgrass cover or quality. Marginally safe chemicals had adverse effects, but treated plots eventually achieved ratings similar to some safe chemicals. Data were subjected to analysis of variance (ANOVA) using the SAS GLM procedure (SAS, 1996), and treatment means were separated at $P \leq 0.05$ by Fisher's protected least significant difference test (LSD) (Steele et al., 1997).

\section{Results and Discussion}

Fall seedling tolerance. Cover and quality were used to assess the effects of the chemicals on seedling tolerance. When the chemicals were applied at 2 weeks after seedling emergence ( 2 WASE) in 1998, data collected on 12 Nov. and 23 Dec. showed that bensulide and siduron did not reduce cover (Table 1). Ethofumesate (16\% to $26 \%$ ) and paclobutrazol (8\% to $12 \%$ ) reduced cover, when compared to the untreated control between 12 Nov. and 23 Dec. Chlorsulfuron was extremely phytotoxic and reduced cover $78 \%$ to $84 \%$ compared to untreated plots during the same time period. Cover ratings increased between 9 Feb. and 8 Apr. 1999, but the same trends were evident. By 8 Apr., ethofumesate (93\% cover) and chlorsulfuron ( $25 \%$ cover)-treated plots had cover ratings that were less than those observed in the control (98\% cover).

Quality ratings for 2 WASE treatments reflected cover ratings (Table 1). Bensulide and siduron-treated plots generally had quality equivalent to the untreated control from 12 Nov. 1998 to 8 Apr. 1999. Quality initially was poor in ethofumesate and paclobutrazol-treated plots, and was extremely poor in chlorsulfurontreated plots. Paclobutrazol-treated bentgrass recovered by spring (17 Mar.). By the final rating date (8 Apr.), ethofumesate and especially chlorsulfuron-treated plots had quality inferior to the control. Hence, bensulide and siduron were safe and paclobutrazol was marginally safe to apply 2 WASE in 1998.

There was much less injury when the chemicals were applied at 4 WASE in 1998. Only chlorsulfuron had reduced cover among the treatments when plots were rated between 12 Nov. 1998 and 17 Mar. 1999 (Table 1). Cover ratings initially (12 Nov. to 9 Feb.) were reduced $9 \%$ to $15 \%$ of the control by chlorsulfuron, but there was good recovery and cover was equivalent to the control by 8 Apr. 1999 .

Table 1. Percent cover and turfgrass quality of 'Crenshaw' creeping bentgrass seedlings treated with five herbicides and paclobutrazol at three timings, $1998-99$.

\begin{tabular}{|c|c|c|c|c|c|c|c|c|c|c|c|c|}
\hline \multirow[b]{3}{*}{ Treatment } & \multirow{3}{*}{$\begin{array}{c}\text { Rate } \\
\left(\mathrm{kg} \cdot \mathrm{ha}^{-1}\right)\end{array}$} & \multirow{3}{*}{$\begin{array}{l}\text { Timing } \\
\text { WASE }^{\mathrm{x}}\end{array}$} & \multicolumn{5}{|c|}{$\operatorname{Cover}^{2}(\%)$} & \multicolumn{5}{|c|}{ Quality $^{\mathrm{y}}(0-10)$} \\
\hline & & & \multicolumn{2}{|c|}{1998} & \multicolumn{3}{|c|}{1999} & \multicolumn{2}{|c|}{1998} & \multicolumn{3}{|c|}{1999} \\
\hline & & & 12 Nov. & 23 Dec. & 9 Feb. & 17 Mar. & 8 Apr. & 12 Nov. & 23 Dec. & 19 Jan. & 17 Mar. & 8 Apr. \\
\hline$\overline{\text { Bensulide }}$ & 8.4 & 2 & $90 a b^{w}$ & $95 \mathrm{ab}$ & $94 \mathrm{a}$ & $97 \mathrm{ab}$ & $97 \mathrm{a}$ & $6.5 \mathrm{~cd}$ & 6.4 cde & $6.4 \mathrm{bcd}$ & $6.8 \mathrm{ab}$ & $6.9 \mathrm{bc}$ \\
\hline Ethofumesate & 0.84 & 2 & $68 d$ & $80 \mathrm{c}$ & $80 \mathrm{~d}$ & $90 \mathrm{~d}$ & $93 \mathrm{~b}$ & $4.4 \mathrm{f}$ & $4.8 \mathrm{f}$ & $4.5 \mathrm{~g}$ & $5.5 \mathrm{e}$ & $5.5 \mathrm{~d}$ \\
\hline Siduron & 6.7 & 2 & 90 abc & $96 \mathrm{a}$ & $95 \mathrm{a}$ & $97 \mathrm{abc}$ & $99 \mathrm{a}$ & $6.6 \mathrm{bcd}$ & $7.0 \mathrm{a}-\mathrm{d}$ & $7.0 \mathrm{ab}$ & $6.8 \mathrm{ab}$ & $7.3 \mathrm{abc}$ \\
\hline Chlorsulfuron & 0.14 & 2 & $10 \mathrm{e}$ & $18 \mathrm{~d}$ & $14 \mathrm{e}$ & $26 \mathrm{e}$ & $25 \mathrm{c}$ & $1.0 \mathrm{~g}$ & $0.8 \mathrm{~g}$ & $0.4 \mathrm{~h}$ & $0.8 \mathrm{f}$ & $0.3 \mathrm{e}$ \\
\hline Paclobutrazol & 0.14 & 2 & $82 \mathrm{c}$ & $88 \mathrm{bc}$ & $86 \mathrm{~cd}$ & $92 \mathrm{bcd}$ & $98 \mathrm{a}$ & $5.1 \mathrm{ef}$ & $5.6 \mathrm{ef}$ & $5.0 \mathrm{fg}$ & 5.9 cde & $6.8 \mathrm{bc}$ \\
\hline Bensulide & 8.4 & 4 & $94 \mathrm{a}$ & $97 \mathrm{a}$ & $97 \mathrm{a}$ & $98 \mathrm{a}$ & $99 \mathrm{a}$ & $7.2 \mathrm{abc}$ & $7.2 \mathrm{abc}$ & 6.2 cde & $7.3 \mathrm{a}$ & $7.6 \mathrm{abc}$ \\
\hline Ethofumesate & 0.84 & 4 & $91 \mathrm{ab}$ & $91 \mathrm{ab}$ & $93 \mathrm{ab}$ & $95 \mathrm{a}-\mathrm{d}$ & $98 \mathrm{a}$ & $6.6 \mathrm{bcd}$ & $6.0 \mathrm{de}$ & 5.5 ef & $6.4 \mathrm{bcd}$ & $7.4 \mathrm{abc}$ \\
\hline Siduron & 6.7 & 4 & $94 \mathrm{a}$ & $98 \mathrm{a}$ & $98 \mathrm{a}$ & $98 \mathrm{a}$ & $99 \mathrm{a}$ & $7.4 \mathrm{abc}$ & $7.0 \mathrm{a}-\mathrm{d}$ & $7.3 \mathrm{a}$ & $7.3 \mathrm{a}$ & $8.4 \mathrm{a}$ \\
\hline Chlorsulfuron & 0.14 & 4 & $84 \mathrm{bc}$ & $81 \mathrm{c}$ & $87 \mathrm{bc}$ & $92 \mathrm{~cd}$ & $96 \mathrm{a}$ & $5.8 \mathrm{de}$ & $4.3 \mathrm{f}$ & $4.8 \mathrm{~g}$ & $5.7 \mathrm{de}$ & $6.3 \mathrm{~cd}$ \\
\hline Paclobutrazol & 0.14 & 4 & $94 \mathrm{a}$ & $96 a$ & $96 \mathrm{a}$ & $97 \mathrm{ab}$ & $99 \mathrm{a}$ & $7.0 \mathrm{abc}$ & $6.8 \mathrm{a}-\mathrm{d}$ & $5.7 \mathrm{def}$ & $6.7 \mathrm{ab}$ & $7.4 \mathrm{abc}$ \\
\hline Ethofumesate & 0.84 & 7 & $-\mathrm{v}$ & $97 \mathrm{a}$ & $97 \mathrm{a}$ & $97 \mathrm{ab}$ & $99 \mathrm{a}$ & - & $7.8 \mathrm{a}$ & $6.8 \mathrm{abc}$ & $7.0 \mathrm{ab}$ & $7.8 \mathrm{ab}$ \\
\hline Prodiamine & 0.36 & 7 & - & $96 \mathrm{a}$ & $96 \mathrm{a}$ & $98 \mathrm{a}$ & $99 \mathrm{a}$ & - & $7.2 \mathrm{abc}$ & $6.5 \mathrm{a}-\mathrm{d}$ & $7.2 \mathrm{ab}$ & $7.8 \mathrm{ab}$ \\
\hline Untreated & - & - & $94 \mathrm{a}$ & $96 a$ & $96 a$ & $97 \mathrm{abc}$ & $98 \mathrm{a}$ & $7.6 \mathrm{abc}$ & $7.5 \mathrm{ab}$ & $6.4 \mathrm{bcd}$ & $6.6 \mathrm{abc}$ & $7.4 \mathrm{abc}$ \\
\hline
\end{tabular}

${ }^{2}$ Percent bentgrass cover was assessed on a $0 \%$ to $100 \%$ linear scale where $0=$ bareground and $100=$ complete bentgrass cover.

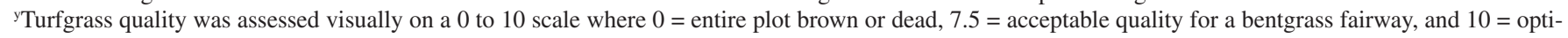
mum greenness and density.

${ }^{x}$ WASE = weeks after seedling emergence; application timings were as follows: 2 WASE on 12 Oct.; 4 WASE on 28 Oct.; and 7 WASE on 17 Nov. 1998.

${ }^{w}$ Means in a column followed by the same letter are not significantly different according to Fisher's protected LSD $(P \leq 0.05)$.

vTreatment not applied until 17 Nov. 1998.

Table 2. Percent cover and turfgrass quality of 'L-93' creeping bentgrass seedlings treated with four herbicides and paclobutrazol at two timings, $2000-01$.

\begin{tabular}{|c|c|c|c|c|c|c|c|c|c|c|c|c|c|}
\hline \multirow[b]{3}{*}{ Treatment } & \multirow{3}{*}{$\begin{array}{c}\text { Rate } \\
\mathrm{kg} \cdot \mathrm{ha}^{-1}\end{array}$} & \multirow{3}{*}{$\begin{array}{l}\text { Timing } \\
\text { WASE }^{\mathrm{x}}\end{array}$} & \multicolumn{5}{|c|}{$\operatorname{Cover}^{2}(\%)$} & \multicolumn{6}{|c|}{ Quality $^{\mathrm{y}}(0-10)$} \\
\hline & & & \multicolumn{3}{|c|}{2000} & \multicolumn{2}{|c|}{2001} & \multicolumn{3}{|c|}{2000} & \multicolumn{3}{|c|}{2001} \\
\hline & & & 19 Oct. & 3 Nov. & 24 Nov. & 27 Feb. & $23 \mathrm{Apr}$. & 19 Oct. & 3 Nov. & 24 Nov. & 27 Dec. & 27 Feb. & $23 \mathrm{Apr}$. \\
\hline$\overline{\text { Bensulide }}$ & 14.0 & 2 & $83 b^{W}$ & $86 \mathrm{~b}$ & $97 \mathrm{a}$ & $96 \mathrm{~b}$ & $89 \mathrm{~d}$ & $4.6 \mathrm{~b}$ & $7.3 \mathrm{~b}$ & $7.1 \mathrm{~b}$ & $5.4 \mathrm{a}$ & $5.6 \mathrm{c}$ & $4.8 \mathrm{~d}$ \\
\hline Ethofumesate & 0.84 & 2 & $29 \mathrm{c}$ & $44 \mathrm{c}$ & $47 \mathrm{~b}$ & $64 c$ & $64 \mathrm{e}$ & $1.9 \mathrm{c}$ & $3.8 \mathrm{~d}$ & $4.3 \mathrm{c}$ & $2.8 \mathrm{~b}$ & $2.1 \mathrm{~d}$ & $2.9 \mathrm{e}$ \\
\hline Siduron & 9.0 & 2 & $96 \mathrm{a}$ & $94 \mathrm{a}$ & $100 \mathrm{a}$ & $100 \mathrm{a}$ & $98 \mathrm{a}$ & $6.8 \mathrm{a}$ & $8.4 \mathrm{a}$ & $8.5 \mathrm{a}$ & $6.0 \mathrm{a}$ & $8.3 \mathrm{a}$ & $7.8 \mathrm{a}$ \\
\hline Prodiamine & 0.36 & 2 & $96 a$ & $90 \mathrm{ab}$ & $100 \mathrm{a}$ & $97 \mathrm{ab}$ & $91 \mathrm{bcd}$ & $6.4 \mathrm{a}$ & $8.0 \mathrm{ab}$ & $7.8 \mathrm{ab}$ & $5.6 \mathrm{a}$ & $6.6 \mathrm{~b}$ & $5.8 \mathrm{c}$ \\
\hline Bensulide & 14.0 & 4 & $-\mathrm{v}$ & $86 \mathrm{~b}$ & $100 \mathrm{a}$ & $100 \mathrm{a}$ & $98 \mathrm{ab}$ & - & $6.3 \mathrm{c}$ & $7.8 \mathrm{ab}$ & $6.0 \mathrm{a}$ & $7.8 \mathrm{a}$ & $8.1 \mathrm{a}$ \\
\hline Ethofumesate & 0.84 & 4 & - & $85 \mathrm{~b}$ & $100 \mathrm{a}$ & $97 \mathrm{ab}$ & $90 \mathrm{~cd}$ & - & $6.3 \mathrm{c}$ & $7.4 \mathrm{~b}$ & $5.6 \mathrm{a}$ & $7.9 \mathrm{a}$ & $6.8 \mathrm{~b}$ \\
\hline Siduron & 9.0 & 4 & - & $91 \mathrm{ab}$ & $97 \mathrm{a}$ & $99 \mathrm{ab}$ & $97 \mathrm{abc}$ & - & $7.9 \mathrm{ab}$ & $7.4 \mathrm{~b}$ & $5.5 \mathrm{a}$ & $7.6 \mathrm{a}$ & $7.5 \mathrm{ab}$ \\
\hline Prodiamine & 0.36 & 4 & - & $94 \mathrm{a}$ & $100 \mathrm{a}$ & $99 \mathrm{ab}$ & $95 \mathrm{a}-\mathrm{d}$ & - & $8.1 \mathrm{a}$ & $7.5 \mathrm{~b}$ & $5.5 \mathrm{a}$ & $7.9 \mathrm{a}$ & $7.8 \mathrm{a}$ \\
\hline Untreated & - & - & 94 a & $94 \mathrm{a}$ & $98 \mathrm{a}$ & $100 \mathrm{a}$ & $98 \mathrm{ab}$ & $6.2 \mathrm{a}$ & $8.4 \mathrm{a}$ & $7.8 \mathrm{ab}$ & $5.7 \mathrm{a}$ & $8.0 \mathrm{a}$ & $8.3 \mathrm{a}$ \\
\hline
\end{tabular}

zPercent bentgrass cover was assessed on a $0 \%$ to $100 \%$ linear scale where $0=$ bareground and $100=$ complete bentgrass cover.

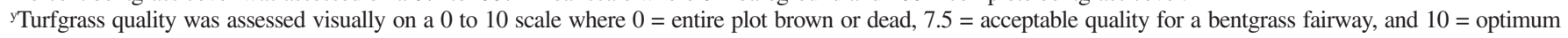
greenness and density.

${ }^{x}$ WASE = weeks after seedling emergence; application timings were as follows: 2 WASE on 6 Oct.; and 4 WASE on 23 Oct. 2000.

"Means in a column followed by the same letter are not significantly different according to Fisher's protected LSD $(P \leq 0.05)$.

vTreatment not applied until 23 Oct. 2000. 
Table 3. Percent cover and turfgrass quality of 'L-93' creeping bentgrass seedlings treated with four herbicides and paclobutrazol in one timing, 2001-02.

\begin{tabular}{|c|c|c|c|c|c|c|c|}
\hline \multirow[b]{3}{*}{ Treatment } & \multirow{3}{*}{$\begin{array}{c}\text { Rate } \\
\mathrm{kg} \cdot \mathrm{ha}^{-1}\end{array}$} & \multirow{3}{*}{$\begin{array}{l}\text { Timing } \\
\text { WASE }^{x}\end{array}$} & \multicolumn{2}{|c|}{$\operatorname{Cover}^{2}(\%)$} & \multicolumn{3}{|c|}{ Quality $^{\mathrm{y}}(0-10)$} \\
\hline & & & \multicolumn{2}{|c|}{2001} & \multicolumn{3}{|c|}{2001} \\
\hline & & & 19 Oct. & 16 Nov. & 26 Oct. & 16 Nov. & 6 Dec. \\
\hline$\overline{\text { Bensulide }}$ & 8.4 & 4 & $92 \mathrm{abc}^{\mathrm{w}}$ & $98 \mathrm{a}$ & $8.3 \mathrm{a}$ & $8.3 \mathrm{ab}$ & $7.8 \mathrm{a}$ \\
\hline Bensulide & 14 & 4 & $91 \mathrm{abc}$ & $98 \mathrm{a}$ & $7.8 \mathrm{ab}$ & $8.1 \mathrm{abc}$ & $8.0 \mathrm{a}$ \\
\hline Siduron & 6.7 & 4 & $87 \mathrm{c}$ & $98 \mathrm{a}$ & $7.1 \mathrm{~b}$ & $7.6 \mathrm{c}$ & $7.9 \mathrm{a}$ \\
\hline Siduron & 9.0 & 4 & $94 \mathrm{ab}$ & $100 \mathrm{a}$ & $7.8 \mathrm{ab}$ & $7.9 \mathrm{bc}$ & $7.8 \mathrm{a}$ \\
\hline Ethofumesate & 0.84 & 4 & $91 \mathrm{abc}$ & $98 \mathrm{a}$ & $7.1 \mathrm{~b}$ & $8.5 \mathrm{a}$ & $8.3 \mathrm{a}$ \\
\hline Ethofumesate & $0.84+0.84$ & 4 & 89 bc & $98 \mathrm{a}$ & $7.3 \mathrm{~b}$ & $8.3 \mathrm{ab}$ & $7.4 \mathrm{a}$ \\
\hline Prodiamine & 0.36 & 4 & $95 \mathrm{a}$ & $98 \mathrm{a}$ & $8.3 \mathrm{a}$ & $8.3 \mathrm{ab}$ & $7.6 \mathrm{a}$ \\
\hline Paclobutrazol & 0.14 & 4 & $92 \mathrm{abc}$ & $98 \mathrm{a}$ & $7.6 \mathrm{ab}$ & $6.1 \mathrm{~d}$ & $7.6 \mathrm{a}$ \\
\hline Untreated & - & - & $93 \mathrm{abc}$ & $98 \mathrm{a}$ & $8.2 \mathrm{a}$ & $8.0 \mathrm{abc}$ & $7.7 \mathrm{a}$ \\
\hline
\end{tabular}

${ }^{2}$ Percent bentgrass cover was assessed on a $0 \%$ to $100 \%$ linear scale where $0=$ bareground and $100=$ complete bentgrass cover.

${ }^{y}$ Turfgrass quality was assessed visually on a 0 to 10 scale where $0=$ entire plot brown or dead, $7.5=$ acceptable quality for a bentgrass fairway, and $10=$ optimum greenness and density.

${ }^{2}$ WASE $=$ weeks after seedling emergence; treatments were applied 4 WASE on 12 Oct.; sequential ethofumesate treatment was applied 5 Nov. 2001.

${ }^{\text {w}}$ Means in a column followed by the same letter are not significantly different according to Fisher's protected LSD $(P \leq 0.05)$.

Turf quality was reduced by ethofumesate on 23 Dec. 1998 and 18 Jan. 1999, when compared to the control. Thereafter, quality of ethofumesate-treated plots was equivalent to the control. Chlorsulfuron-treated plots exhibited reduced quality on all dates between 12 Nov. 1998 and 17 Mar. 1999, but ratings were equivalent to the control by 8 Apr.

Prodiamine was not applied until 7 WASE. Both ethofumesate and prodiamine applied at this time did not reduce cover or quality on any date. Chlorsulfuron was marginally safe to apply 4 WASE; whereas, all other chemicals were judged to be safe to apply at either 4 or 7 WASE.

In 2000, the chemicals were applied either 2 or 4 WASE. The bensulide rate was increased from 8.4 to $14.0 \mathrm{~kg} \cdot \mathrm{ha}^{-1}$ and the siduron rate was increased from 6.7 to 9.0 $\mathrm{kg} \cdot \mathrm{ha}^{-1}$ in 2000. Chlorsulfuron was deleted as a treatment because it was generally too phytotoxic. Paclobutrazol was not evaluated in 2000. Ethofumesate greatly reduced cover within 2 weeks (19 Oct.) following the 2 WASE application and plots never achieved a level of cover equivalent to the control (Table 2). In the 2 WASE application of bensulide, cover was reduced when compared to the control on 19 Oct. and 3 Nov., but plots recovered and achieved cover equivalent to the control by 24 Nov. However, by Feb. 27, and as late as the final rating (23 Apr.), cover again declined in bensulide-treated plots and cover was reduced 9\% when compared to the control. Siduron and prodiamine-treated plots had cover ratings equivalent to the control on all rating dates. Bensulide (except 24 Nov. and 27 Dec.) and especially ethofumesate-treated plots had quality inferior to the control on all dates. Prodiamine did not reduce quality in 2000 , but plots exhibited a reduction in quality by spring 2001. Siduron-treated plots had quality ratings equivalent to the control on all rating dates. Except for siduron, all herbicides were judged to be too injurious to apply 2 WASE in 2000.

Bensulide and ethofumesate applied in the 4 WASE timing initially reduced cover $8 \%$ to $9 \%$, when compared to the control (Table 2). Plots treated with the aforementioned herbicides, however, achieved complete cover by 24 Nov. Cover ratings for ethofumesate-treated plots, however, declined by 23 Apr. 2001. Siduron and prodiamine-treated plots had cover equivalent to the control on all rating dates. Turf quality of bensulide and ethofumesate-treated plots was reduced initially on 3 Nov., but was equivalent to the control by 24 Nov. 2000. On the final rating date (23 Apr.), however, the quality of ethofumesate-treated bentgrass had declined and was inferior to the control. These results indicated that bensulide, siduron and prodiamine generally were safe to apply at 4 WASE. Ethofumesate was injurious, but was judged to be marginally safe to apply 4 WASE.

Because only siduron and bensulide (8.4 $\mathrm{kg} \cdot \mathrm{ha}^{-1}$ in 1998) were safe to apply 2 WASE in the first 2 study years, treatments only were applied 4 WASE in 2001. Two rates of three herbicides and one rate of paclobutrazol and prodiamine were reevaluated in the final year. None of the treatments reduced cover when compared to the control in 2001 and by 16 Nov. all treated plots had between $98 \%$ and $100 \%$ cover (Table 3). Plots treated with siduron at $6.7 \mathrm{~kg} \cdot \mathrm{ha}^{-1}$, however, initially had lower cover ratings when compared to plots treated with siduron $\left(9.0 \mathrm{~kg} \cdot \mathrm{ha}^{-1}\right)$ and prodiamine.

Two weeks following treatment (26 Oct.),

Table 4. Soil residual effect of five herbicides and paclobutrazol applied in Fall 1998 ('Crenshaw') or 2000 ('L-93') on creeping bentgrass cover in Spring 1999 or 2001; WASE = weeks after seedling emergence.

\begin{tabular}{|c|c|c|c|c|c|c|c|c|c|}
\hline \multirow[b]{3}{*}{ Treatment } & \multirow{3}{*}{$\begin{array}{c}\text { Rate } \\
\left(\mathrm{kg} \cdot \mathrm{ha}^{-1}\right)\end{array}$} & \multirow{3}{*}{$\begin{array}{l}\text { Timing } \\
\text { WASE }\end{array}$} & \multicolumn{7}{|c|}{$\operatorname{Cover}^{2}(\%)$} \\
\hline & & & \multicolumn{3}{|c|}{$1999^{y}$} & \multicolumn{4}{|c|}{$2001^{x}$} \\
\hline & & & 20 May & 3 June & 17 June & 15 May & 29 May & 12 June & 19 June \\
\hline$\overline{\text { Bensulide }}$ & 8.4 & 2 & $27 \mathrm{de}^{\mathrm{w}}$ & $78 \mathrm{ab}$ & $95 \mathrm{a}$ & $-^{v}$ & - & - & - \\
\hline Bensulide & 14.0 & 2 & - & - & - & $27 \mathrm{~d}$ & $57 \mathrm{c}$ & $84 \mathrm{~cd}$ & $96 \mathrm{ab}$ \\
\hline Ethofumesate & 0.84 & 2 & $47 \mathrm{a}$ & $87 \mathrm{a}$ & $96 \mathrm{a}$ & $55 \mathrm{a}$ & $84 \mathrm{a}$ & $97 \mathrm{a}$ & $100 \mathrm{a}$ \\
\hline Siduron & 6.7 & 2 & $35 \mathrm{a}-\mathrm{e}$ & $83 \mathrm{ab}$ & $96 a$ & - & - & - & - \\
\hline Siduron & 9.0 & 2 & - & - & - & $44 \mathrm{bc}$ & $66 \mathrm{bc}$ & $90 \mathrm{bc}$ & $98 \mathrm{a}$ \\
\hline Chlorsulfuron & 0.14 & 2 & $47 \mathrm{a}$ & $86 \mathrm{a}$ & $95 \mathrm{a}$ & - & - & - & - \\
\hline Prodiamine & 0.36 & 2 & - & - & - & 7 ef & $10 \mathrm{e}$ & $36 \mathrm{e}$ & $65 \mathrm{c}$ \\
\hline Paclobutrazol & 0.14 & 2 & $43 \mathrm{ab}$ & $86 \mathrm{a}$ & 99 a & - & - & - & - \\
\hline Bensulide & 8.4 & 4 & $31 \mathrm{~b}-\mathrm{e}$ & $83 \mathrm{ab}$ & $97 \mathrm{a}$ & - & - & - & - \\
\hline Bensulide & 14.0 & 4 & - & - & - & $15 \mathrm{e}$ & $40 \mathrm{~d}$ & $80 \mathrm{~d}$ & $91 \mathrm{~b}$ \\
\hline Ethofumesate & 0.84 & 4 & $39 \mathrm{a}-\mathrm{d}$ & $83 a b$ & $97 \mathrm{a}$ & $51 \mathrm{ab}$ & $69 \mathrm{~b}$ & $94 \mathrm{ab}$ & $100 \mathrm{a}$ \\
\hline Siduron & 6.7 & 4 & $28 \mathrm{c}-\mathrm{e}$ & $82 \mathrm{ab}$ & $97 \mathrm{a}$ & - & - & - & - \\
\hline Siduron & 9.0 & 4 & - & - & - & $33 \mathrm{~cd}$ & $57 \mathrm{c}$ & $92 \mathrm{ab}$ & $98 \mathrm{a}$ \\
\hline Chlorsulfuron & 0.14 & 4 & $24 \mathrm{e}$ & $73 \mathrm{~b}$ & $93 a$ & - & - & - & - \\
\hline Prodiamine & 0.36 & 4 & - & - & - & $3 \mathrm{f}$ & $7 \mathrm{e}$ & $22 \mathrm{f}$ & $48 \mathrm{~d}$ \\
\hline Paclobutrazol & 0.14 & 4 & $42 \mathrm{abc}$ & $87 \mathrm{a}$ & $99 \mathrm{a}$ & - & - & - & - \\
\hline Ethofumesate & 0.84 & 7 & 38 a-e & $85 \mathrm{a}$ & $97 \mathrm{a}$ & - & - & - & - \\
\hline Prodiamine & 0.36 & 7 & $8 \mathrm{f}$ & $46 \mathrm{c}$ & $79 b$ & - & - & - & - \\
\hline Untreated & - & - & $34 \mathrm{a}-\mathrm{e}$ & $86 \mathrm{a}$ & $98 \mathrm{a}$ & $50 \mathrm{ab}$ & $65 \mathrm{bc}$ & $91 \mathrm{ab}$ & $98 \mathrm{a}$ \\
\hline
\end{tabular}

${ }^{2}$ Percent bentgrass cover was assessed on a $0 \%$ to $100 \%$ linear scale where $0=$ bareground and $100=$ complete bentgrass cover.

${ }^{y}$ Site was treated with glyphosate 23 Apr. 1999 and disk-seeded 6 May 1999. Application timings were as follows: 2 WASE on 12 Oct.; and 4 WASE on 28 Oct.; and 7 WASE on 17 Nov. 1998.

${ }^{x}$ Site was treated with glyphosate 23 Apr. 2001 and disk-seeded 1 May 2001. Application timings were as follows: 2 weeks after seedling emergence (2 WASE) on 6 Oct.; 4 WASE on 23 Oct. 2000.

${ }^{\text {w} M e a n s ~ i n ~ a ~ c o l u m n ~ f o l l o w e d ~ b y ~ t h e ~ s a m e ~ l e t t e r ~ a r e ~ n o t ~ s i g n i f i c a n t l y ~ d i f f e r e n t ~ a c c o r d i n g ~ t o ~ F i s h e r ' s ~ p r o t e c t e d ~ L S D ~}(P \leq 0.05)$.

vreatment not evaluated. 
Table 5. Soil residual effects of four herbicides and paclobutrazol applied in Fall 2001 on 'L-93' creeping bentgrass cover in Spring 2002.

\begin{tabular}{|c|c|c|c|c|c|c|}
\hline \multirow[b]{2}{*}{ Treatment } & \multirow{2}{*}{$\begin{array}{c}\text { Rate } \\
\left(\mathrm{kg} \cdot \mathrm{ha}^{-1}\right)\end{array}$} & \multirow{2}{*}{$\begin{array}{l}\text { Timing } \\
\text { WASE }^{y}\end{array}$} & \multicolumn{4}{|c|}{$\operatorname{Cover}^{2}(\%)$} \\
\hline & & & 31 May & 6 June & 24 June & 18 July \\
\hline Bensulide & 8.4 & 4 & $21 \mathrm{a}^{\mathrm{x}}$ & $31 \mathrm{ab}$ & $89 \mathrm{a}$ & $99 \mathrm{a}$ \\
\hline Bensulide & 14.0 & 4 & $13 \mathrm{ab}$ & $17 \mathrm{bc}$ & $70 \mathrm{~b}$ & $88 \mathrm{~b}$ \\
\hline Siduron & 6.7 & 4 & $25 \mathrm{a}$ & $34 \mathrm{ab}$ & $87 \mathrm{a}$ & $98 \mathrm{a}$ \\
\hline Siduron & 9.0 & 4 & $19 \mathrm{a}$ & $36 \mathrm{ab}$ & $89 \mathrm{a}$ & 99 a \\
\hline Ethofumesate & 0.84 & 4 & $22 \mathrm{a}$ & $45 \mathrm{a}$ & $92 \mathrm{a}$ & $100 \mathrm{a}$ \\
\hline Ethofumesate & $0.84+0.84$ & 4 & $21 \mathrm{a}$ & $43 \mathrm{a}$ & $94 \mathrm{a}$ & $100 \mathrm{a}$ \\
\hline Prodiamine & 0.36 & 4 & $2 \mathrm{~b}$ & $3 c$ & $20 \mathrm{c}$ & $53 \mathrm{c}$ \\
\hline Paclobutrazol & 0.14 & 4 & $18 \mathrm{a}$ & $33 \mathrm{ab}$ & $89 \mathrm{a}$ & $99 \mathrm{a}$ \\
\hline Untreated & - & - & $24 \mathrm{a}$ & $48 \mathrm{a}$ & $94 \mathrm{a}$ & $99 \mathrm{a}$ \\
\hline
\end{tabular}

${ }^{2}$ Percent bentgrass cover was assessed on a $0 \%$ to $100 \%$ linear scale where $0=$ bareground and $100=$ complete bentgrass cover.

${ }^{y}$ WASE = weeks after seedling emergence; treatments were applied on 12 Oct. 2001; sequential ethofumesate treatment was applied 5 Nov. and the site was overseeded 10 May 2002.

${ }^{x}$ Means in a column followed by the same letter are not significantly different according to Fisher's protected LSD $(P \leq 0.05)$.

siduron $\left(6.7 \mathrm{~kg} \cdot \mathrm{ha}^{-1}\right)$ and ethofumesate (both rates) had reduced bentgrass quality, when compared to the control. Plots treated the second time with ethofumesate on 5 Nov. did not sustain any reduction in quality. $\mathrm{Pa}$ clobutrazol-treated plots had poorest quality among all treatments on 16 Nov. Data collected between 6 Dec. 2001 and 22 Apr. 2002 showed that none of the treatments had a long-term adverse effect on bentgrass quality (all data not shown). Therefore, all herbicide rates and paclobutrazol were judged to be safe to apply 4 WASE in the final study year.

Soil residual effects. In the spring following the fall application of chemicals each study site was treated with glyphosate and renovated to determine if the fall applied chemicals would have any adverse soil residual effects on seedling emergence and establishment. Only percent bentgrass cover ratings were used as a measure of establishment in the spring reseeding portion of the study. The 1998-99 site was reseeded on 6 May 1999. By 20 May, only plots treated with prodiamine (7 WASE; 17 Nov. 1998) had exhibited a reduction in seedling emergence and therefore cover, when compared to all other treatments (Table 4). The adverse effects of prodiamine soil residues were evident on all rating dates, and on the final rating date (17 Jun.) cover was reduced $19 \%$ when compared to the control. Chlorsulfuron (4 WASE; 28 Oct. 1998) overseeded plots also exhibited a reduction in cover on 20 May and 3 June, when compared to several other herbicides. However, on the final rating date (17 June), all chemical-treated plots (except prodiamine) had cover ratings equivalent to the control. Hence, only soil residues of prodiamine and possibly chlorsulfuron were sufficient to adversely affect a spring overseeding with bentgrass.

In 2001, the site was reseeded on 1 May. Bentgrass cover data collected 15 May showed that the prodiamine residues ( 2 and 4 WASE) again had greatly reduced seedling emergence and therefore cover (Table 4). Bensulide (14.0 $\mathrm{kg} \cdot \mathrm{ha}^{-1}$ ) applied 23 Oct. 2000 had resulted in a reduction of cover that was greater than the same treatment applied 6 Oct. 2000. Similarly, plots treated with siduron $\left(9.0 \mathrm{~kg} \cdot \mathrm{ha}^{-1}\right)$ on 23 Oct., but not 6 Oct., exhibited a reduction in cover when compared to the control. Ethofume- sate did not adversely impact bentgrass seedling emergence. Cover ratings increased between 15 May and 12 June, but reductions in cover again were observed in plots of the previously noted treatments, except for siduron applied 23 Oct. By 19 June, only plots treated with prodiamine (both timings) and bensulide (applied 23 Oct.) had cover ratings inferior to the control. Hence, prodiamine severely reduced establishment. Bensulide applied in the later (23 Oct.), but not in the earlier (6 Oct.) timing also adversely affected establishment.

In 2002, two rates of bensulide, siduron and ethofumesate were evaluated as well as one rate of prodiamine and paclobutrazol. The site was reseeded on 10 May. From 31 May to the final rating date (18 July), prodiamine-treated plots had less cover than all other chemicaltreated plots (Table 5). A reduction in cover became evident in plots treated with the high rate of bensulide $\left(14.0 \mathrm{~kg} \cdot \mathrm{ha}^{-1}\right)$ on 6 Jun. Plots treated with the high rate of bensulide did not achieve cover levels equivalent to the control by the end of the study. Both rates of siduron and ethofumesate, and paclobutrazol did not adversely influence cover.

Due to variation in treatments and planting dates among years, differences between creeping bentgrass cultivars (L-93 and Crenshaw) were not determined. Both cultivars, however, exhibited similar effects from each herbicide and paclobutrazol treatment. This study showed that siduron $\left(6.7\right.$ and $\left.9.0 \mathrm{~kg} \cdot \mathrm{ha}^{-1}\right)$ and bensulide $\left(8.4 \mathrm{~kg} \cdot \mathrm{ha}^{-1}\right)$ were safe to apply to bentgrass seedlings 2 WASE. Bensulide $\left(14.0 \mathrm{~kg} \cdot \mathrm{ha}^{-1}\right)$, prodiamine and paclobutrazol were noninjurious to seedlings when applied 4 WASE. Ethofumesate $\left(0.84 \mathrm{~kg} \cdot \mathrm{ha}^{-1}\right)$ caused a reduction in cover and quality when applied 4 WASE, but a second fall application in 2001 resulted in no additional injury. Furthermore, ethofumesate soil residuals did not adversely affect reseeding. Hence, in a situation where there is severe competition between annual bluegrass and bentgrass seedlings it may be prudent to consider using ethofumesate to improve the competitiveness of the bentgrass despite some potential injury. Applications of bensulide $\left(8.4 \mathrm{~kg} \cdot \mathrm{ha}^{-1}\right)$ or siduron 2 WASE, and bensulide $\left(14.0 \mathrm{~kg} \cdot \mathrm{ha}^{-1}\right)$ or prodiamine $(0.36$ $\left.\mathrm{kg} \cdot \mathrm{ha}^{-1}\right) 4$ WASE also may offer a potential for reducing annual bluegrass competition in bentgrass seedlings. The potential soil residual risks of using prodiamine, however, are great. Should fall bentgrass establishment fail, prodiamine soil residuals would likely preclude a successful spring renovation. Long soil persistence of prodiamine was previously observed by Fermanian and Haley (1994). They reported that prodiamine ( 0.8 to $\left.2.2 \mathrm{~kg} \cdot \mathrm{ha}^{-1}\right)$ applied in the fall reduced Kentucky bluegrass (Poa pratensis $\mathrm{L}$.) seedling emergence for up to 488 days after the herbicide was last applied. The overwintering soil residue of bensulide (14.0 $\mathrm{kg} \cdot \mathrm{ha}^{-1}$ ) also was shown to reduce bentgrass establishment significantly in this study, but it was not nearly as detrimental as prodiamine. Applying paclobutrazol to seedlings 4 WASE does not injure bentgrass seedlings. It remains to be determined if paclobutrazol would help or hinder bentgrass seedling establishment in the presence of competition from annual bluegrass. Indeed, the next phase of study would involve the use of these chemicals at sites where there is a mix of annual bluegrass and creeping bentgrass seedlings.

\section{Literature Cited}

Branham, В. 1991. Dealing with Poа аппиа. Golf Course Mgt. 59(9):46, 48, 52, 54, 58, 60.

Callahan, L.M. and E.R. McDonald. 1992. Effectiveness of bensulide in controlling two annual bluegrass (Роа аппиа) subspecies. Weed Technol. 6:97-103.

Christians, N. 1996. A historical perspective of annual bluegrass control. Golf Course Mgt. 64(11):49-57.

Dernoeden, P.H. 1998. Use of prodiamine as a preemergence herbicide to control annual bluegrass in kentucky bluegrass. HortScience 33:845-846.

Dernoeden, P.H. and T.R. Turner. 1988. Annual bluegrass control and tolerance of kentucky bluegrass and perennial ryegrass to ethofumesate. HortScience 23:565-567.

Fermanian, T.W. and J.E. Haley. 1994. Application of prodiamine to immature turf. Weed Technol. 8:617-620.

Gaul, M.C. and N.E. Christians. 1988. Selective control of annual bluegrass in cool-season turfs with fenarimol and chlorsulfuron. Agron. J. 80:120-125.

Goss, R.L., T.W. Cook, S.E. Brauen, and S.P. Orton. 1980. Effects of repeated applications of bensulide and tricalcium arsenate on the control of annual bluegrass and on quality of Highland colonial bentgrass putting green turf. Proc. 3rd Intl. Turfgrass Res. Conf. 3:247-255.

Jagschitz, J. 1979. Annual bluegrass control in kentucky bluegrass with herbicides. Proc. N.E. Weed Sci. Soc. 33:308-313.

Johnson, B.J. and T.R. Murphy. 1995. Effect of paclobutrazol and flurprimidol on suppression of Poа апnиа spp. reptans in creeping bentgrass (Agrostis stolonifera) greens. Weed Technol. 9: 182-186.

Maloy, B.M. and N.E. Christians. 1986. Chlorsulfuron activity on cool-season grasses. HortScience 21:1012-1014.

SAS Institute. 1996. SAS user's guide: Statistics. version 6.11. SAS Inst., Cary, N.C.

Steele, R.G.D., J.H. Torrie, and D.A. Dickey. 1997. Principles and procedures of statistics: A biometrical approach. $3^{\text {rd }}$ ed. McGraw-Hill, Inc., New York. 